EPJ Web of Conferences 70, 00012 (2014)

DOI: $10.1051 /$ epjconf/ 20147000012

(C) Owned by the authors, published by EDP Sciences, 2014

\title{
Incurable Adler relation for soft neutrino interactions
}

\author{
B.Z. Kopeliovich ${ }^{\mathrm{a}}$, I.K. Potashnikova ${ }^{\mathrm{b}}$, Iván Schmidt ${ }^{\mathrm{c}}$, and M. Siddikov ${ }^{\mathrm{d}}$ \\ Departamento de Física, Universidad Técnica Federico Santa María, \\ Centro de Estudios Subatómicos, and Centro Científico-Tecnológico de Valparaíso, \\ Casilla 110-V, Valparaíso, Chile
}

\begin{abstract}
The Adler relation (AR), which bridges soft interactions of neutrinos and pions, might look as a manifestation of pion dominance. However neutrino cannot fluctuate to a pion because of lepton current conservation, instead it interacts via much heavier hadronic components. This fact leads to nontrivial relations between interaction amplitudes of different hadronic species, in particular, it links diagonal and off-diagonal diffractive interactions of pions. Absorptive corrections break these relations making the AR impossible to hold universally, i.e. for any target and at any energy. We predict a dramatic breakdown of the AR for coherent neutrino-production of pions on nuclei at all energies.
\end{abstract}

\section{Introduction}

In 1964 Stephen Adler derived the eminent relation [1] between the cross sections of forward neutrinoto-lepton scattering and pion cross section on the same hadronic target with the same final hadronic state. Although it is tempting to interpret such a relation as pion pole dominance, it was realized [2-4] that this is not correct. Neutrino cannot fluctuate into a pion, $v \rightarrow l+\pi$, because of the transversity of the lepton current. Such a transition is completely forbidden for neutral currents and suppressed by the lepton mass squared for charge current processes. The meaning of the Adler relation (AR) is more delicate. Hadronic fluctuations of a neutrino are much heavier than the pion (even heavier than the $\rho$-meson), but miraculously the neutrino cross section mediated by such heavy fluctuations turns out to be related to the pion interaction. The underlying dynamics of such a link is unknown, as well as the mechanism of chiral symmetry breaking. It is based on the phenomenon of partial conservation of axial current (PCAC). We demonstrate here that strong absorptive corrections on nuclear targets cause a dramatic suppression of diffractive neutrino-production of pions compared with the expectations based on the AR.

\section{PCAC}

In the chiral limit of massless quarks both the vector and axial currents are conserved:

$$
\begin{aligned}
& q_{\mu} V_{\mu}=q_{\mu}\left[\bar{q}\left(k^{\prime}\right) \gamma_{\mu} q(k)\right]=0 ; \\
& q_{\mu} A_{\mu}=q_{\mu}\left[\bar{q}\left(k^{\prime}\right) \gamma_{5} \gamma_{\mu} \tau q(k)\right]=0,
\end{aligned}
$$

where $q_{\mu}=k_{\mu}^{\prime}-k_{\mu}$.

\footnotetext{
${ }^{a}$ e-mail: boris.kopeliovich@usm.cl

b e-mail: irina.potashnikova@usm.cl

c e-mail: ivan.schmidt@usm.cl

d e-mail: marat.siddikov@usm.cl
}

This is an Open Access article distributed under the terms of the Creative Commons Attribution License 2.0, which permits unrestricted use, distribution, and reproduction in any medium, provided the original work is properly cited. 
Hadrons acquire large masses via the mechanism of spontaneous symmetry breaking. The hadronic currents may be still conserved. For the vector current this is rather obvious:

$$
q_{\mu} j_{\mu}^{V}=q_{\mu} \bar{p}\left(k^{\prime}\right) \gamma_{\mu} n(k)=\left(m_{n}-m_{p}\right) \bar{p} n=0,
$$

up to the QED corrections.

Conservation of axial current looks more involved:

$$
q_{\mu} j_{\mu}^{A}=q_{\mu} \bar{p}\left(k^{\prime}\right) \gamma_{\mu} \gamma_{5} n(k)=\left(m_{n}+m_{p}\right) \bar{p} \gamma_{5} n \neq 0 .
$$

Nevertheless, in the general form,

$$
j_{\mu}^{A}=\bar{p}\left(k^{\prime}\right)\left[g_{A} \gamma_{\mu} \gamma_{5}-g_{p} q_{\mu} \gamma_{5}\right] n(k),
$$

the axial current can be conserved if

$$
g_{P}\left(Q^{2}\right)=g_{A}\left(Q^{2}\right) \frac{2 m_{N}}{Q^{2}} .
$$

The pole behavior here shows presence of a massless Goldstone particle [5]. This proves the Goldstone theorem: spontaneous breaking of chiral symmetry generates massless particles identified with pions.

\subsection{Goldberger-Treiman conspiracy}

PCAC leads to a miraculous relation between the quantities having very different origins.

$$
\sqrt{2} m_{N} g_{A}(0)=f_{\pi} g_{\pi N N}
$$

where $f_{\pi}$ and $g_{\pi N N}$ are the the pion decay and pion-nucleon couplings, respectively, both known from data. It is tempting to interpret this Goldberger-Treiman relation [6] in terms of pion pole dominance, like is illustrated by the first term in the left panel of Fig. 1. However, the pion pole does not contribute


Fig. 1. Left: graphical representation for the effective pseudo-scalar and axial-vector couplings in Eq. (4). Right: fake analogy between pion pole and $\rho$-meson dominance in production of hadronic states $X$ in lepton-proton interactions.

to $\beta$-decay, because the lepton current is conserved (up to the electron mass), $\Gamma(\pi \rightarrow \bar{v} e) \propto m_{e}^{2}$.

The axial-vector formfactor $g_{A}\left(Q^{2}\right)$ represents the contribution of heavy states, which are related to the pion term via PCAC. John Bell called this relation between the axial constant and the pion pole Goldberger-Treiman conspiracy [2]. 


\subsection{Hadronic properties of neutrinos}

Although the non-trivial Goldberger-Treiman relation is well confirmed by data for neutron decay and muon capture, the PCAC hypothesis should be tested thoroughly in other processes.

The Fock components of a high-energy neutrino at low scale are dominated by the axial-vector hadronic fluctuations, since the vector term vanishes at $Q^{2} \rightarrow 0$ due to CVC. The amplitude of the process $v+p \rightarrow l+X$, where $X$ is the final hadronic state, has the form,

$$
M=\frac{G}{\sqrt{2}} l_{\mu}\left(V_{\mu}+A_{\mu}\right)
$$

where the conserved lepton current reads,

$$
l_{\mu}=\bar{l}\left(k^{\prime}\right) \gamma_{\mu}\left(1+\gamma_{5}\right) v(k)
$$

At $Q^{2} \rightarrow 0$ the vector current contribution and the transverse part of the axial term vanish, only $\sigma_{L}^{A}$ survives, correspondingly, the matrix element squared has the factorized form,

$$
|\bar{M}|^{2}=\frac{G^{2}}{2} L_{\mu \nu} A_{\mu \nu},
$$

where the lepton tensor $L_{\mu \nu}$ in the limit $Q^{2} \rightarrow 0$ reads,

$$
L_{\mu \nu}\left(Q^{2} \rightarrow 0\right)=2 \frac{E_{v}\left(E_{v}-v\right)}{v^{2}} q_{\mu} q_{v}
$$

Here $E_{v}$ is the energy of the neutrino, and $v=E_{v}-E_{l}$ is the transferred energy in the target rest frame. Remarkably, this tensor is proportional to $q_{\mu} q_{v}$, so one can apply the PCAC relation in the form [4],

$$
q_{\mu} j_{\mu}^{A}=m_{\pi}^{2} \phi_{\pi}
$$

which leads to the Adler relation (AR),

$$
\left.\frac{d^{2} \sigma(v p \rightarrow l X)}{d Q^{2} d v}\right|_{Q^{2}=0}=\frac{G^{2}}{2 \pi^{2}} f_{\pi}^{2} \frac{E_{v}-v}{E_{v} v} \sigma(\pi p \rightarrow X) .
$$

In analogy to the vector dominance model it is tempting to interpret the Adler relation as a manifestation of pion dominance, as is illustrated in Fig. 1 (right panel).

However, neutrinos do not fluctuate to pions because of conservation of the lepton current, $q_{\mu} l_{\mu}=0$ in Eq. (7), so the pion intermediate state does not contribute, i.e. the above interpretation of the AR is not correct.

To understand the physics of the AR, let us single out the main singularities in the dispersion relation for the axial current amplitude,

$$
A_{\mu}\left(Q^{2}\right)=\frac{f_{\pi} q_{\mu}}{Q^{2}+m_{\pi}^{2}} T(\pi p \rightarrow X)+\frac{f_{a_{1}}}{Q^{2}+m_{a_{1}}^{2}} T_{\mu}\left(a_{1} p \rightarrow X\right)+\ldots
$$

The first term, the pion pole, does not contribute to the process due to conservation of the lepton current, $q_{\mu} l_{\mu}=0$. So the intermediate hadronic states must be heavier axial-vector hadrons, e.g. the $a_{1}$ meson, which is the chiral partner of the $\rho$-meson. It seems to be natural to expect $a_{1}$ dominance in the axial current, in analogy with the vector meson dominance. In this case one can restrict the dispersion relation for the axial current by the first two terms, explicitly shown in Eq. (13). Then these terms must be related in order to cancel in $q_{\mu} A_{\mu}$ and provide PCAC, eq. (11).

Such a nontrivial relation between the two terms in (13) was challenged by Piketty and Stodolsky [3], who found that the AR leads to the equality $\sigma_{\text {diff }}\left(\pi p \rightarrow a_{1} p\right) \approx \sigma_{e l}(\pi p \rightarrow \pi p)$, which contradicts data by factor $20(!)$. 
The problem is relaxed after inclusion of the $\rho \pi$ cut and other diffractive excitations into the dispersion relation $[4,7]$. Indeed, the relation

$$
\sigma_{\text {diff }}(\pi p \rightarrow X p) \approx \sigma_{e l}(\pi p \rightarrow \pi p)
$$

does not contradict data. The $\rho \pi$ cut can be represented by an effective pole $\tilde{a}_{1}[7,8]$, because the invariant mass distribution in the $1^{+} S$ wave in diffractive dissociation $\pi p \rightarrow 3 \pi p$ indeed demonstrates a rather narrow peak shown in the left panel of Fig. 2. This bump is usually related to the non-resonance
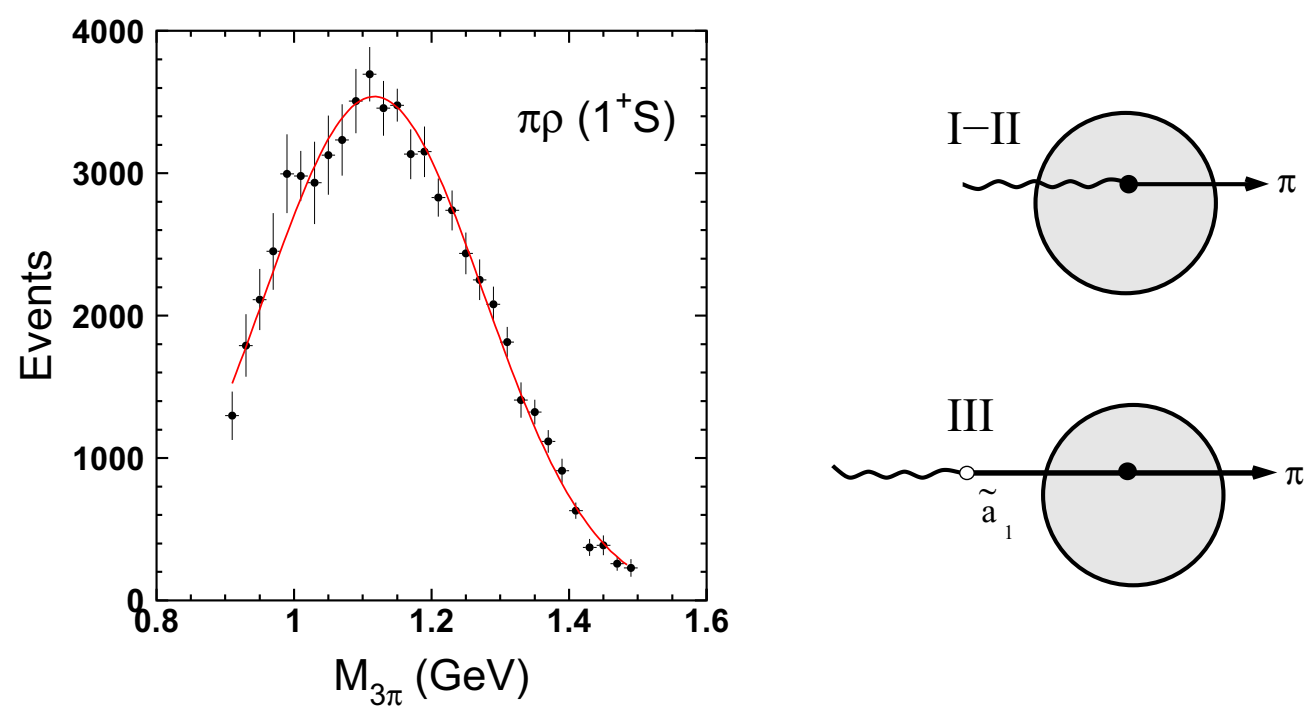

Fig. 2. Left: Invariant mass distribution of $3 \pi$ produced diffractively as $1^{+} S$ wave in $\pi p \rightarrow \pi \rho p$. Right: two regimes for coherent pion neutrino-production off nuclei. The upper and the lower figures correspond to the regimes described in the text, I-II and III respectively. the waving line shows the axial current, $W$ or $Z$ bosons fluctuating to light hadronic states.

Deck mechanism [9]. Thus, we arrive at the two-pole $\left(\pi+\tilde{a}_{1}\right)$ model [10].

\section{Absorptive corrections}

\subsection{Incurable AR}

Although the (approximate) equality (14) seems to cure the striking controversy between the AR and data, this cannot be an universal remedy for any target and at any energy. As an example, one can check the relation (14) in the Froissart regime expected to onset at very high energies. In this regime $\sigma_{e l}(\pi p \rightarrow \pi p) \rightarrow \sigma_{\text {tot }} / 2$, while $\sigma_{\text {diff }}(\pi p \rightarrow X p) \propto \sigma_{\text {tot }} / \ln (s)$. This happens because diffraction is suppressed by absorptive corrections, while elastic cross section is enhanced. Thus, the condition (14) is badly broken in this regime.

Another way to approach the black-disc regime of interaction is to use nuclear targets, for which the AR predicts $\sigma_{\text {diff }}^{\pi A} \approx \sigma_{e l}^{\pi A}$. However, such a relation should be severely broken because nuclear absorption enhances elastic, but suppresses diffractive interactions, so $\sigma_{e l}^{\pi A} \propto A^{2 / 3}$, but $\sigma_{\text {diff }}^{\pi A} \propto A^{1 / 3}$.

Thus, the AR is incurable: even if the relation (14) were valid for some target and at some energy, it will certainly fail for heavy nuclei, or/and at very high energies. The diffractive diagonal and offdiagonal amplitudes cannot be universally related, since they are affected by absorptive corrections differently. 


\subsection{Time scales for diffractive neutrino-nucleus interactions}

In what follows we are going to test the $\mathrm{AR}$ in the process of diffractive pion production by neutrinos on proton, or nuclear targets, e.g. $v+p \rightarrow l+\pi+p$. Within the considered two-channel model $\left(\pi+\tilde{a}_{1}\right)$ the neutrino-nucleus interactions are characterized by two time scales:

$$
\begin{aligned}
t_{c}^{\pi} & =\frac{2 v}{m_{\pi}^{2}+Q^{2}} \\
t_{c}^{\tilde{a}_{1}} & =\frac{2 v}{Q^{2}+m_{\tilde{a}_{1}}^{2}} .
\end{aligned}
$$

The pion coherence time controls the interferences between pions produced at different longitudinal coordinates $[7,11-13]$. The second time scale can be interpreted as the $\tilde{a}_{1}$ fluctuation lifetime [13, 10]. Apparently, at small $Q^{2} \ll m_{\tilde{a}_{1}}^{2}$ these time scales are very different, $t_{c}^{\pi} \gg t_{c}^{\tilde{a}_{1}}$. Depending on the transferred energy $v=E_{v}-E_{l}$ one can identify three different regimes of diffractive neutrino-nucleus interactions:

I. $v<\left(Q^{2}+m_{\pi}^{2}\right) R_{A}$, which corresponds to rather low energies, approximately $v \lesssim 500 \mathrm{MeV}$. In this regime the nuclear formfactor $F_{A}\left(q_{L}^{2}\right)$, where $q_{L}=1 / t_{c}^{\pi}$, strongly suppresses the coherent production, which leaves the nucleus intact. Correspondingly, the cross section is steeply falling with the atomic number as $A^{2} \exp \left[-R_{A}^{2} / 3\left(t_{c}^{\pi}\right)^{2}\right]$, where $R_{A} \propto A^{1 / 3}$ is the nuclear radius.

II. $\left(Q^{2}+m_{\pi}^{2}\right) R_{A}<v<\left(Q^{2}+m_{\tilde{a}_{1}}^{2}\right) R_{A}$, what approximately corresponds to the energy range $0.5 \lesssim$ $v \lesssim 40 \mathrm{GeV}$. In this regime the absorptive corrections are caused by final-state interactions of the produced pion, while shadowing effects are still small. The cross section rises with $A$ as $A^{2 / 3}$.

III. $v>\left(Q^{2}+m_{\tilde{a}_{1}}^{2}\right) R_{A}$, what approximately corresponds to energies $v>40 \mathrm{GeV}$. This is the regime of maximal shadowing corrections [14]. Neutrino fluctuates to a hadronic state, $v \rightarrow l+\tilde{a}_{1}$, which diffractively produces a pion, $\tilde{a}_{1}+A \rightarrow \pi+A$. In this case only the periphery of the nucleus contributes, so the cross section is $\propto A^{1 / 3}$.

The nuclear effects for the cross section of coherent neutrino-production of pions are usually characterized by the ratio,

$$
R_{A / N}^{c o h}\left(v, Q^{2}\right)=\frac{d \sigma(v A \rightarrow l \pi A) / d Q^{2} d v}{A d \sigma(v N \rightarrow l \pi N) / d Q^{2} d v}
$$

The results of calculations [10] within the two-channel model are shown in Fig. 3. Comparison with the horizontal dashed lines, which present the expectations based on the AR, indeed demonstrates existence of three energy regimes for the nuclear effects. At low energies, regime I, the AR is trivially broken due to large longitudinal momentum transfer, which does not exist in pion-nucleus elastic scattering. In the regime II the validity of the AR is surprisingly restored. The corresponding energy range is rather wide, $v$ varies by an order of magnitude. However at high energies in the regime III the AR is severely broken by strong absorptive corrections. The cross section is below the AR prediction by factor $A^{1 / 3}$, which is a large number for heavy nuclei.

\subsection{Dipole representation}

An extension of two channels to a multi-channel model would unavoidably lead to a large number of adjusted parameters. Instead, we switch to the dipole representation, which effectively includes all intermediate states in all orders [15]. In this case pion production can be described as $v p \rightarrow l(\bar{q} q) p \rightarrow$ $l \pi p$, i.e. neutrino fluctuates into a $\bar{q} q$ dipole, which interacts elastically with the target and then is projected to the pion wave function [16]. This process is illustrated in the graph in the left panel of Fig. 4 . The cross section of this process has the form,

$$
v \frac{d^{3} \sigma_{v p \rightarrow \mu \pi p}}{d v d t d Q^{2}}=\frac{G_{F}^{2} L_{\mu v}\left(W_{\mu}^{A}\right)^{*} W_{v}^{A}}{32 \pi^{3} m_{N}^{2} E_{v}^{2} \sqrt{1+Q^{2} / v^{2}}},
$$



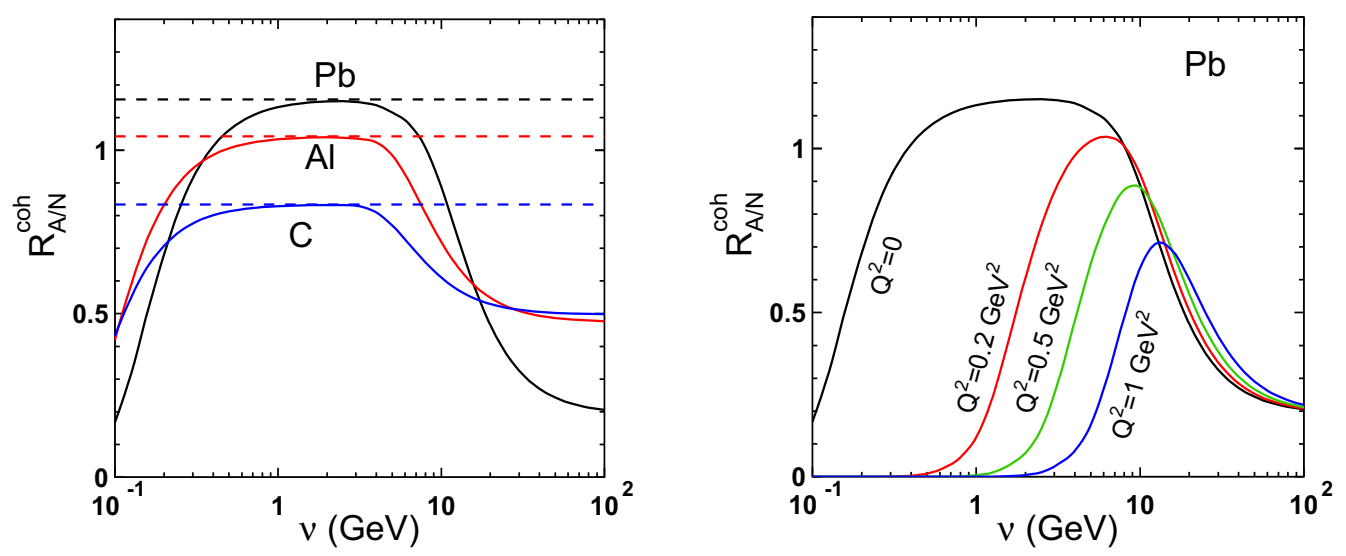

Fig. 3. Left: nuclear ratio Eq. (16) at $Q^{2}=0$ as function of the transferred energy $v$ for carbon, aluminum and lead. Dashed line show the expectations based on the AR. Solid curves show the results of calculations with the two-channel model [10]. Right: the same as in the left figure, but only for lead and at different virtualities $Q^{2}$.
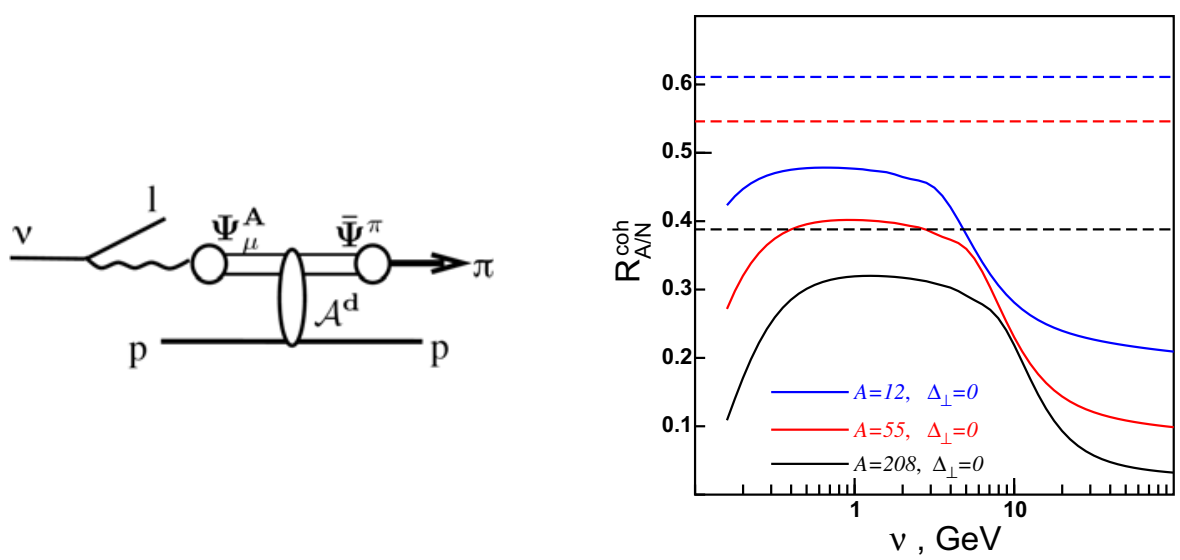

Fig. 4. Left: the hadronic fluctuation of the neutrino is a $\bar{q} q$ pair, which interacts elastically and then is projected into the pion light-cone wave function. Right: The results of calculation within the dipole representation [17], plotted by solid curves, are compared with the AR predictions, shown by horizontal dashed lines.

where

$$
W_{\mu}^{A}\left(s, \Delta, Q^{2}\right)=\int_{0}^{1} d \beta d^{2} r \bar{\Psi}^{\pi}(\beta, r) \mathcal{A}^{d}(\beta, r ; \Delta) \Psi_{\mu}^{A}(\beta, r) .
$$

Here $\mathcal{A}^{d}(\beta, r ; \Delta)$ is the dipole amplitude, which depends on dipole separation $r$, fractional light-cone momentum $\beta$ of $q$ or $\bar{q}$, and transverse momentum $\Delta$. This amplitude is pretty well known from phenomenology, since it has been fitted to photoproduction and DIS data. The light-cone $\bar{q} q$ distribution amplitudes $\Psi_{\mu}^{A}$ and $\Psi^{\pi}$ are calculated in the instanton vacuum model [18-20] (see details in[16,21]).

The results for nuclear effects are shown by solid curves in the right panel of Fig. 4 in comparison with the AR predictions plotted by dashed lines. Differently from the results of the two-channel approximation, Fig. 3, now the cross section is quite below the AR benchmark at all energies, and it is easy to understand why. Within the two-channel approximation, the two time scales Eqs. (16) are well defined, and all events within the certain energy range (see Fig. 3) belong to the regime II, where the $\mathrm{AR}$ is valid. On the contrary, the invariant mass of the dipole is not fixed, but varies with integration 
over $\beta$ and $r$. The low mass tail of the dipoles can be associated with a long coherence time $t_{c}^{d i p} \gg t_{c}^{\tilde{a}_{1}}$ which also gets into the regime III, where the nuclear ratio is strongly suppressed (see Fig. 3). As a result, the nuclear ratio plotted in Fig. 4 is significantly below the level imposed by the AR.

\subsection{Incoherent diffractive neutrino-production of pions on nuclei}

In a diffractive process the target nucleus can be excited and decay to fragments without new particle production. Such a channel has a significant fractional cross section and is usually called incoherent production. In this case the nuclear ration is defined similar to (16).

$$
R_{A / N}^{i n c o h}\left(v, Q^{2}\right)=\frac{d \sigma\left(v A \rightarrow l \pi A^{*}\right) / d Q^{2} d v}{A d \sigma(v N \rightarrow l \pi N) / d Q^{2} d v}
$$

The nuclear effects for incoherent production $v+A \rightarrow l+\pi+A^{*}$ have different assignments for the regimes considered above. The regime I does not exist, because the nucleus breaks up and there is no nuclear formfactor suppressing the cross section. So the regime II starts at very low energies, and its atomic number dependence is $A^{2 / 3}$. This is much higher than the cross section calculated with the $\mathrm{AR}$, which links this incoherent process to quasielastic pion-nucleus scattering. The latter is know to be significantly suppressed, as $A^{1 / 3}$ [22]. Thus, the AR grossly underestimate the incoherent neutrinoproduction of pions at low energies.

However at higher energies, in the regime III the incoherent cross section drops to $A^{1 / 3}$, because both the $\tilde{a}_{1}$ and pion are subject to absorption, and the two-channel model exactly reproduces the result of the AR [10]. The transition between the regimes II and III depends on $Q^{2}$ and is shown for lead in the left panel of Fig. 5. We see that at $Q^{2}=0$ the AR is restored at $v>1 \mathrm{GeV}$.
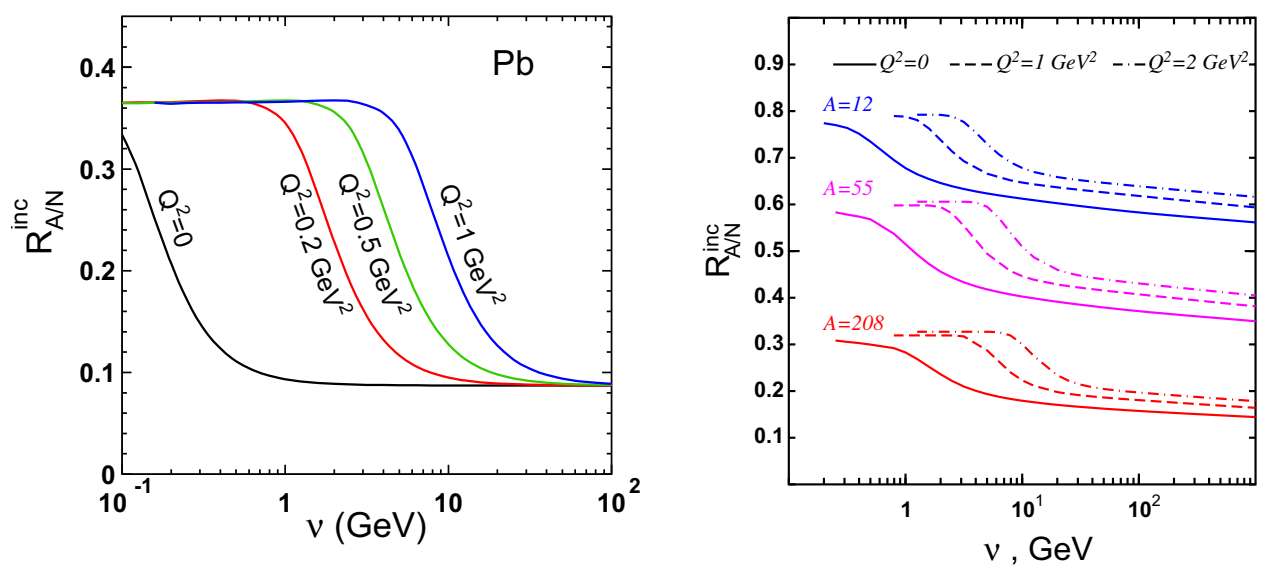

Fig. 5. The nuclear ratio Eq. (19) for incoherent neutrino-production of pions on nuclei as function of energy. Left: The results of the two-channel approximation for lead at different virtualities $Q^{2}$. Right: The results of the dipole approach.

The dipole approach leads to similar results, much closer to the AR in the regime III than for coherent production in the regime II. The reason is clear: in spite of the low-mass tail of the dipoles, which leads to a longer coherence time, the process remains in the same regime III. The results of the dipole approach are shown for several nuclei in the right panel of Fig. 5. 


\section{Summary}

- The Goldberger-Treiman relation is not a result of pion exchange, which is suppressed in $\beta$-decay and muon capture. This is a result of a miraculous link between light and heavy states.

- In the diffractive neutrino-production of pions PCAC establishes a link between diagonal and offdiagonal amplitudes, which cannot be correct, because both are strongly and differently affected by absorption.

- The Adler relation for coherent neutrino-production of pions is always broken, but especially at high energies. On the contrary, in incoherent pion production the Adler relation is broken at low, but is restored at high energies.

Acknowledgements: B.Z.K. is thankful to the organizers of the International Conference on New Frontiers in Physics for the invitation to deliver this talk. Our work was supported in part by Fondecyt (Chile) grants 1090291, 1090236, 1100287 and 1120920.

\section{References}

1. S. L. Adler, Phys. Rev. 135, (1964) B963.

2. J. S. Bell, In Cumming, J. (ed.): Osborn, H. (ed.): Hadron interactions of electrons and photons, Academic Press, (1971) 369-394

3. C. A. Piketty and L. Stodolsky, Nucl. Phys. B 15, (1970) 571

4. B. Z. Kopeliovich and P. Marage, Int. J. Mod. Phys. A 8, (1993) 1513

5. J. Goldstone, A. Salam and S. Weinberg, Phys. Rev. 127, (1962) 965

6. M. L. Goldberger and S. B. Treiman, Phys. Rev. 110, (1958) 1178

7. A. A. Belkov and B. Z. Kopeliovich, Sov. J. Nucl. Phys. 46, (1987) 499 [Yad. Fiz. 46, (1987) 874]

8. B. Z. Kopeliovich, I. K. Potashnikova, I. Schmidt and J. Soffer, Phys. Rev. D 84, (2011) 114012

9. R. T. Deck, Phys. Rev. Lett. 13, (1964) 169

10. B. Z. Kopeliovich, I. K. Potashnikova, I. Schmidt and M. Siddikov, Phys. Rev. C 84, (2011) 024608

11. B. Z. Kopeliovich, Phys. Lett. B 227, (1989) 461

12. B. Z. Kopeliovich, Sov. Phys. JETP 70, (1990) 801 [Zh. Eksp. Teor. Fiz. 97, (1990) 1418]

13. B. Z. Kopeliovich, Nucl. Phys. Proc. Suppl. 139, (2005) 219

14. B. Z. Kopeliovich, J. G. Morfin and I. Schmidt, arXiv:1208.6541 [hep-ph]; to appear in "Progress in Particle and Nuclear Physics" (2012)

15. B.Z. Kopeliovich, L.I. Lapidus and A.B. Zamolodchikov, Sov. Phys. JETP Lett. 33, (1981) 595 [Pisma v Zh. Exper. Teor. Fiz. 33, (1981) 612]

16. B. Z. Kopeliovich, I. Schmidt and M. Siddikov, Phys. Rev. D 84, (2011) 033012

17. B. Z. Kopeliovich, I. Schmidt and M. Siddikov, Phys. Rev. D 85, (2012) 073003

18. T. Schäfer and E. V. Shuryak, Rev. Mod. Phys. 70 (1998) 323 [arXiv:hep-ph/9610451]

19. D. Diakonov and V. Y. Petrov, Nucl. Phys. B 272, (1986) 457

20. D. Diakonov, M. V. Polyakov and C. Weiss, Nucl. Phys. B 461, (1996) 539 [arXiv:hep$\mathrm{ph} / 9510232]$

21. B. Z. Kopeliovich, I. Schmidt and M. Siddikov, arXiv:1108.5654 [hep-ph]

22. B. Z. Kopeliovich, I. K. Potashnikova and I. Schmidt, Phys. Rev. C 73, (2006) 034901 